\title{
Enregistrement des recherches dans des registres publics: point de la situation
}

\section{Myriam Rège-Walther, Bernard Burnand}

Cochrane Suisse Institut universitaire de médecine sociale et préventive (IUMSP), CHUV et Université de Lausanne

\section{Correspondance:}

Myriam Rège-Walther

Cochrane Suisse

Unité d'évaluation des soins Institut universitaire de

médecine sociale et préventive (IUMSP)

CHUV

Bugnon 17

CH-1005 Lausanne

myriam.rege@chuv.ch

www.swiss.cochrane.org

\section{La situation actuelle}

De la transparence, s'il vous plaît! C'est la revendication d'un nombre croissant d'individus, d'organisations et d'institutions publiques qui utilisent les résultats de la recherche médicale pour des décisions de soins. Ce besoin de transparence fait suite au constat de manipulation, masquage ou divulgation partielle de résultats d'essais cliniques conduits par l'industrie pharmaceutique, qui ont eu des conséquences directes en termes de santé et de coûts, tant sur des patients que sur des collectivités publiques. Si l'ensemble des résultats des recherches avait été publié, la sécurité des patients concernés aurait été mieux protégée. La récente polémique relayée par le BMJ sur l'efficacité réelle de l'oseltamivir (Tamiflu ${ }^{\circledR}$ ) pour prévenir les complications de la grippe illustre bien cette problématique [1]. Ainsi, les résultats de huit essais cliniques menés par Roche, mais non publiés, ont été inclus dans une revue systématique, qui concluait à l'efficacité du Tamiflu pour réduire les complications dues à la grippe chez les adultes [2]. Les résultats de ces huit essais n'ont pas pu être obtenus pour mettre à jour une revue systématique Cochrane portant sur l'efficacité des inhibiteurs de la neuraminidase pour prévenir et traiter la grippe chez les adultes habituellement en bonne santé [3]. La revue systématique Cochrane conclut, sans avoir pu inclure ces huit essais, qu'il n'y a pas de preuves suffisantes pour affirmer que le Tamiflu est efficace pour prévenir les complications dues à la grippe chez les adultes habituellement en bonne santé. Maintenant, face à ces résultats discordants et au manque de transparence de Roche, la question reste posée: le Tamiflu est-il efficace pour prévenir les complications dues à la grippe chez les adultes?

Plusieurs initiatives ont vu le jour au cours de la dernière décennie pour répondre à ce problème et tenter d'augmenter la transparence dans la recherche clinique. L'Organisation Mondiale de la Santé (OMS), le Comité International des Editeurs de Journaux Médicaux (ICMJE), et l'Association Médicale Mondiale (AMM), notamment, incitent les chercheurs à enregistrer leurs essais cliniques dans un registre publiquement accessible et à rendre compte des résultats de ces essais de manière complète et en respectant des délais raisonnables de publication. Un tel enregistrement permet en effet d'identifier et de prévenir les biais dus à la publication incomplète ou à la non-publication de résultats et d'identifier les essais à mener pour compléter les études déjà effectuées, permettant ainsi de mieux planifier la recherche clinique, de décider des priorités et d'éviter des duplications, en connaissance cause. En outre, nous soulignons la nécessité éthique de transparence vis-à-vis des patients, qui s'engagent volontairement et avec altruisme dans les études, et qui attendent que les résultats des essais auxquels ils ont participé soient rendus publics.

L'OMS a ainsi mis sur pied depuis 2005 le Système d'enregistrement international des essais cliniques (ICTRP) [4]. Ce système a pour but de faciliter la saisie de données descriptives de base des essais, selon une liste développée par l'OMS, et de les rendre publiques. Une plateforme permet de chercher les essais cliniques [5] qui sont répertoriés dans des registres primaires et partenaires, répondant à des critères spécifiques de l'OMS. Le système d'enregistrement de l'OMS fonctionne sur une base volontaire et ne comporte aucune obligation légale.

Depuis 2007, le Comité International des Editeurs de Journaux Médicaux (ICMJE [6]) exige que tout essai clinique voulant faire l'objet d'une publication dans l'un des journaux partenaires, soit enregistré dans un registre publiquement accessible, avant l'enrôlement du premier patient. Cette incitation n'est toutefois contraignante que pour les chercheurs qui souhaitent publier le résultat de leur recherche, ce qui n'est pas forcément le cas de tous les investigateurs d'essais cliniques.

L'Association Médicale Mondiale (AMM), auteure de la Déclaration d'Helsinki qui traite des principes éthiques applicables à la recherche médicale sur l'être humain, en a révisé en 2008 le Principe 19: «Tout essai clinique doit être enregistré dans une base de données accessible au public avant que ne soit recruté le premier sujet de recherche» [7]. Là encore, il ne s'agit nullement d'une obligation légale mais plutôt d'une recommandation éthique.

Ces initiatives ont remporté un certain succès, avec plus de 20000 essais cliniques répondant aux exigences du ICMJE [8] enregistrés début 2009, pour moins de 3000 en 2004.

Cependant bon nombre d'essais cliniques menés pour tester l'efficacité des médicaments n'apparaissent jamais, ou que partiellement, dans les registres ouverts au public. De ce fait, il est impossible de savoir combien d'essais cliniques sont menés dans le monde, quelle est la part des essais à laquelle le public n'a pas accès, ni sur quels aspects de recherche portent ces essais. 
Les résultats indésirables ne doivent pas être tenus secrets.

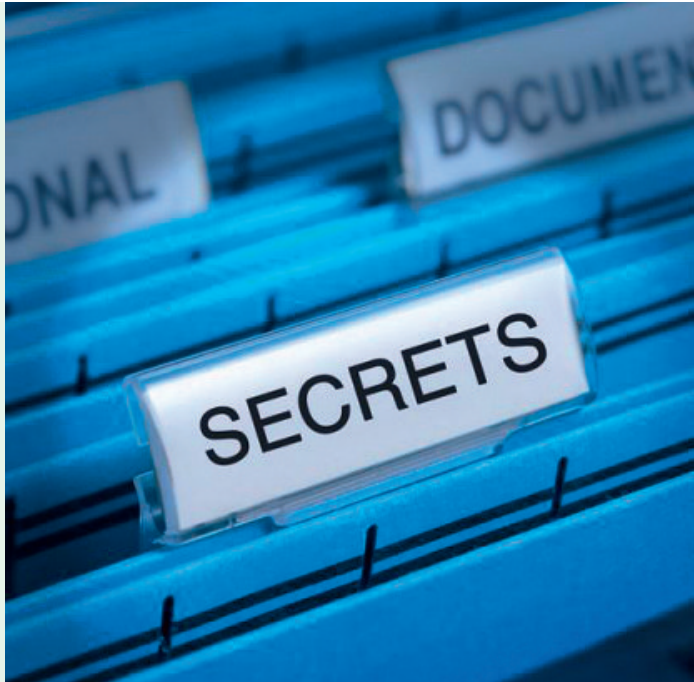

\section{Quel futur?}

Une solution? Elle pourrait consister, d'une part, à augmenter la part de financement public dans la recherche, afin de permettre d'évaluer des interventions qui n'ont que peu d'intérêt pour des développeurs privés et, d'autre part, à édicter des lois obligeant les promoteurs à publier les protocoles de recherche, la recherche en cours ainsi que ses résultats dans des registres publics, sous une forme compréhensible et répondant à un minimum d'exigences, telles celles préconisées par l'OMS. Ces deux options, contribueraient à accroître la transparence.

En Suisse, la législation actuelle n'oblige pas l'enregistrement des essais cliniques dans des registres ouverts. Swissmedic, l'autorité subordonnée au Département fédéral de l'Intérieur, chargée de l'autorisation de mise sur le marché, du contrôle de la fabrication et de la qualité ainsi que de la surveillance du marché des produits thérapeutiques, possède un registre des essais cliniques conduits avec des médicaments, qui n'est pas accessible au public.

Il y a cependant des raisons d'être optimiste. En effet, le 7 mars 2010, le peuple suisse a approuvé l'article $118 \mathrm{~b}$ de la Constitution, base constitutionnelle à la loi fédérale relative à la «Recherche sur l'être humain». L'article 55 du projet de loi mentionne explicitement que la Confédération tient un registre public des projets de recherche autorisés et des résumés des résultats, et que le Conseil fédéral édicte des dispositions sur les données devant figurer dans le registre.

Récemment, en date du 10 mars 2011, le Conseil National a approuvé la loi relative à la recherche sur l'être humain dans son ensemble. Cependant, l'article 55 a été modifié et le terme «projets de recherche autorisés» a été remplacé et restreint par celui d'«études cliniques interventionnelles».

La Commission de la science, de l'éducation et de la culture du Conseil des Etats s'est également penchée sur le projet de loi fin mars 2011, mais elle a ajourné son vote sur l'ensemble. «S'agissant de l'enregistre- ment obligatoire des projets de recherche (art. 55), la commission a approuvé, par 12 voix contre 0 , une nouvelle formulation qui maintient cette obligation, mais qui permet au Conseil fédéral de définir des exceptions en se fondant sur les réglementations internationales reconnues.» [9].

La balle est maintenant dans le camp du Conseil des Etats qui débattra de ce projet de loi, vraisemblablement durant la session d'été 2011.

A l'issue de ces débats parlementaires, notre pays possédera peut-être, et dans un avenir proche, une législation garantissant l'accès à des informations transparentes et de qualité sur les essais cliniques terminés, en cours et à venir. La communauté médicale, les institutions publiques et les patients bénéficieront alors d'une aide précieuse pour prendre des décisions dans le domaine de la santé en meilleure connaissance de cause.

\section{Références}

1 Cohen D. Complications: tracking down the data on oseltamivir. BMJ. 2009; 339:b5387.

2 Kaiser L, Wat C, Mills T, Mahoney P, Ward P, Hayden F. Impact of oseltamivir treatment on influenzarelated lower respiratory tract complications and hospitalizations. Arch Intern Med. 2003;163:1667-72.

3 Jefferson T, Jones MA, Doshi P, Del Mar CB, Dooley L, Hama R, Heneghan CJ. Neuraminidase inhibitors for preventing and treating influenza in healthy adults. Cochrane Database of Systematic Reviews. 2006; Issue 3. Art. No.: CD001265. Doi: 10.1002/14651858.CD001265.pub2.

4 Organisation mondiale de la santé; international clinical trials registry platform, search portal, http://apps.who.int/trialsearch/ (site visité en décembre 2010).

5 Définition de l'essai clinique selon l'OMS: Pour les fins de l'enregistrement, un essai clinique est toute recherche dans laquelle les participants ou les groupes de participants sont affectés, dès le départ, à une ou des interventions d'ordre sanitaire afin d'évaluer les effets de ces dernières sur la santé. Les essais cliniques peuvent aussi être appelés des essais interventionnels. Les interventions portent, entre autres, sur les médicaments, les cellules et autres produits biologiques, les actes chirurgicaux, les techniques radiologiques, les dispositifs, les thérapies comportementales, les changements dans les protocoles de soins, les soins préventifs, etc. Cette définition englobe les Phases d'essai de I à IV.

6 International Committee of Medical Journal Editors. www.icmje.org/ (site visité en décembre 2010).

7 Association médicale mondiale. Déclaration d'Helsinki de L'AMM - Principes éthiques applicables à la recherche médicale impliquant des êtres humains. www.wma.net/fr/30publications/10policies/ b3/index.html (site visité en décembre 2010).

8 Ghersi D, Pang T. From Mexico to Mali: four years in the history of clinical trial registration. 2009; Journal of Evidence-Based Medicine. 2:1-7. doi: 10.1111/j.1756-5391.2009.01014.x

9 Communiqué de presse CSEC-E: Loi relative à la recherche sur l'être humain: la commission au diapason du Conseil fédéral (25/03/2011). 